Pacific Journal of Mathematic 


\section{DIVISORIAL COMPLETE INTERSECTIONS}

\section{JAMES HORNELL}

A complete intersection in a commutative ring $R$ with identity is a regular element $f$ of $R$ such that $g \in R$ and $g / f \in I R$ (the integral closure of $R$ in its total quotient ring) imply that $g / f \in R$. It assumed that $R$ is nonimbedded and that $I R$ is a noetherian $R$-module, and it is proven that the set of complete intersections in $R$ is the set of regular elements of $R$ not contained in any of a certain finite set of prime ideals of $R$, the nonnormal divisorial prime ideals of $R$ together with the prime ideals which occur as an imbedded prime ideal of a proper principal ideal of $R$. This finite set of prime ideals contains the associated prime ideals of the conductor of $I R$ in $R$, but it is shown that this is not always an equality.

In $\S \S 1-5$ are developed properties of zero divisors relative to normalization and extensions of well known properties of integral domains in order to obtain the description of the set of complete intersections in a ring with zero divisors. The associated prime ideals of a proper principal ideal are then described, the set of complete intersections is shown to be closed, and some examples are given. Ring will mean throughout a non-null commutative ring containing an identity.

1. Valuations on a commutative ring. Let $R$ be a commutative ring with identity, and let $G$ be an ordered abelian group written additively. A valuation of $R$ into $G$ is a function $v: R \rightarrow G \cup\{\infty\}$ for which $v(\alpha \beta)=v(\alpha)+v(\beta), v(\alpha+\beta) \geqq \min \{v(\alpha), v(\beta)\}, v(0)=\infty$, and if $v(\alpha)=\infty$ then $\alpha$ is a zero divisor in $R$ for all $\alpha, \beta \in R$ (where $\infty+a=\infty, \infty+\infty=\infty, \infty>a$ for all $a \in G)$. With proof as usual, $v(1)=0, v(-\alpha)=v(\alpha)$, and if $v(\alpha)<v(\beta)$ then $v(\alpha+\beta)=v(\alpha)$.

Let $M$ be a multiplicative subset of $R$, let $\psi: R \rightarrow M^{-1} R$ be the canonical homomorphism, and let $w$ be a valuation of $M^{-1} R$. Then $w \psi$ is a valuation of $R$. Conversely, let $v$ be a valuation of $R$ with $v(M) \subset G$, and define $w(\psi \alpha / \psi \beta)=v(\alpha)-v(\beta)$ for all $a \in R, \beta \in M$. Then $w$ is a valuation of $M^{-1} R$. This defines a bijective correspondence between the valuations of $M^{-1} R$ and the valuations $v$ of $R$ for which $v(M) \subset G$. Let $Q R$ denote the total quotient ring of $R$, and let $I R$ denote the integral closure of $R$ in $Q R$. Thus there is a natural bijective correspondence between the valuations of $R$, those of $Q R$, and those of $I R$.

A valuation $v$ of $R$ is finite on $R$ if $v(\alpha) \geqq 0$ for all $\alpha \in R$. If $v$ is finite on $R$, then the extension of $v$ to $I R$ is finite on $I R$. If $v$ is 
finite on $R$, define the center of $v$ in $R$ to be the prime ideal $\{\alpha \in R \mid v(\alpha)>0\}$.

Let $v$ be a valuation of $R$. Let $N=\{\alpha \in R \mid v(\alpha)=\infty\}$, a prime ideal of $R$ containing only zero divisors. Let $\phi: R \rightarrow R / N$ and $\psi: R / N$ $\rightarrow Q(R / N)$ be the canonical homomomorphisms, and let $K_{v}$ denote $Q(R / N) . v$ extends uniquely to a valuation of $K_{v}, \psi \phi \alpha / \psi \phi \beta \rightarrow v(\alpha+N)-$ $v(\beta+N)$, also denoted by $v$ (where $v \psi \phi$ is the valuation $v$ of $R$ ). The valuation ring of $v, R_{v}$ with maximal ideal $\mathfrak{m}_{v}$ is defined to be the valuation ring of $v$ in $K_{v}$. Let $N_{v}=\{\alpha \in Q R \mid v(\alpha)=\infty\}$.

2. Zero prime ideals, Let $R$ be a commutative ring with identity. A zero prime ideal of $R$ is a prime ideal of $R$ which contains only zero divisors of $R$. The zero prime ideals of $R$, of $Q R$, and of $I R$ are in a bijective correspondence. If $N$ is a zero prime ideal of $R$, let $Q N=(Q R) N$, and let $I N=Q N \cap I R$. A maximal zero prime ideal of $R$ is a maximal element in the set of all zero prime ideals of $R$. Every zero divisor of $R$ is contained in a zero prime ideal of $R$. $R$ is nonimbedded if every zero prime ideal of $R$ is minimal. A minimal prime ideal is a zero prime ideal ([1]). If $R$ is nonimbedded then $I\left(R_{\Re}\right)=R_{\Re}(I R)$ for every prime ideal $\mathfrak{P}$ of $R$ ([1], Cor. 2).

Proposition 1. Let $N$ be a zero prime ideal of $R$. If the injection $j: Q R / Q N \rightarrow Q(R / N)(j:(\alpha / \beta+Q N) \rightarrow(\alpha+N) /(\beta+N))$ is surjective, then $N$ is a maximal zero prime ideal of $R$.

Proposition 2. Let $R$ have only a finite number of maximal zero prime ideals, and let $N$ be one. The injection $j: Q R / Q N \rightarrow Q(R / N)$ is surjective, and $j$ induces an isomorphism $I R / I N \simeq I(R / N)$.

Proof. Let $\alpha+N \in R / N$ be regular. Let $N_{1}=N, N_{2}, \cdots, N_{t}$ be the maximal zero prime ideals of $R$, where $\alpha \notin N_{1} \cup \cdots \cup N_{s}$ and $\alpha \in N_{s+1} \cap \cdots \cap N_{t}$. Let $\beta \in N_{1} \cap \cdots \cap N_{s}$ and $\beta \notin N_{s+1} \cup \cdots \cup N_{t} \cdot \alpha+\beta$ is regular in $R, \alpha+\beta \in \alpha+N$, and $j$ is surjective. $j(I R / I N) \subset I(R / N)$. If $(\alpha+N) /(\beta+N) \in I(R / N)$, then the representative $\beta$ can be chosen to be regular in $R$, and for some $m \in N, a_{i} \in R$

$$
\left(\frac{\alpha}{\beta}\right)^{n}(1+m)+\left(\frac{\alpha}{\beta}\right)^{n-1} a_{n-1}+\cdots+a_{0}=0 .
$$

Thus $\alpha(1+m) / \beta \in I R$ and $j: I R / I N \rightarrow I(R / N)$ is surjective.

Proposition 3. Let $R$ be a nonimbedded ring with a finite number, $n$, of zero prime ideals. Then $I R \simeq R_{1} \oplus \cdots \oplus R_{n}$ where each ring $R_{i}$ has a unique zero prime ideal and $R_{i}$ is the integral closure 
of $R / A_{i}$ for $A_{i}=\left(R_{1}+\cdots+R_{i-1}+R_{i+1}+\cdots+R_{n}\right) \cap R$.

Proof. Let $N_{1}, \cdots, N_{n}$ be the zero prime ideals of $R$. Let $\alpha_{i} \in \bigcap_{j \neq i} N_{j}$ and $\alpha_{i} \notin N_{i}$ for $i=1, \cdots, n$, with $\alpha_{i} \alpha_{j}=0$ for $i \neq j$. $\alpha=\alpha_{1}+\cdots+\alpha_{n}$ is a regular element of $R$. Let $\beta_{i}=\alpha_{i} / \alpha$. Now $\beta_{i}^{2}=\beta_{i}$ so $\beta_{i} \in I R, \beta_{i} \beta_{j}=0$ for $i \neq j$, and $\beta_{i}+\cdots+\beta_{n}=1$. Let $R_{i}=(I R) \beta_{i}$. If $R_{i}$ had more than one zero prime ideal, $R$ would have at least $n+1$ zero prime ideals. $R_{1}+\cdots+R_{i-1}+R_{i+1}+\cdots+$ $R_{n} \subset I N_{i}$, so $A_{i} \subset N_{i} \cdot Q R \simeq Q R_{1} \oplus \cdots \oplus Q R_{n}$. $A_{i}=R \cap\left(Q R_{1}+\cdots+\right.$ $\left.Q R_{i-1}+Q R_{i+1}+\cdots+Q R_{n}\right)$ a contracted ideal, thus $I A_{i}=(Q R \cdot A) \cap$ $I R=R_{1}+\cdots+R_{i-1}+R_{i+1}+\cdots+R_{n}$, and $A_{i} \not \subset N_{j}$ for $i \neq j$. Now, by a proof entirely similar to the proof of Proposition 2, the injection $Q\left(R / A_{i}\right) \rightarrow Q R / Q R \cdot A_{i}$ is surjective and induces an isomorphism $I\left(R / A_{i}\right) \simeq$ $I R / I A_{i} \simeq R_{i}$.

Let $\Re$ be the nil radical of $R$, let $Q \Re$ be the nil radical of $Q R$, and note that $Q \Re \subset I R$.

Proposition 4. Let $R$ be a noetherian, nonimbedded ring. Then the injection $i: Q R / Q \mathfrak{i} \rightarrow Q(R / \mathfrak{N})(i:(\alpha / \beta+Q \mathfrak{R}) \rightarrow(\alpha+\mathfrak{N}) /(\beta+\mathfrak{i}))$ is surjective, and $i$ iduces an isomorphism $I R / I \Re \simeq I(R / \Re)$.

The Proof is similar to the proof of Proposition 2.

3. Prime divisors. Let $v$ be a discrete rank one valuation of $R$, finite on $R$. Let $\Re$ be the center of $v$ in $R$, and let $N=N_{v} \cap R$. $v$ is of the first kind on $R$ if $N$ is a minimal prime ideal of $R$ and $\mathfrak{P} / N$ is of height one in $R / N$. Such a discrete rank one valuation, finite on $R$ of the first kind will be called a prime divisor of $R$.

Proposition 5. Let $R$ be nonimbedded noetherian ring such that $I R / Q \Re$ is a noetherian $R$ module. If $\alpha \in Q R$ and if $v(\alpha) \geqq 0$ for every prime divisor $v$ of $R$, then $\alpha \in I R$.

Proof. $I(R / \Re) \simeq I R / Q \Re$ (Prop. 4) is a noetherian $R$ module. Let $N_{1}, \cdots, N_{t}$ be the zero prime ideals of $R . \quad I(R / \mathfrak{R}) \simeq \bigoplus_{i=1}^{t} I(R / \mathfrak{N}) / I\left(N_{i} / \mathfrak{R}\right)$ and $Q(R / \mathfrak{N})=\bigoplus_{i=1}^{t} Q(R / \mathfrak{N}) / Q\left(N_{i} / \mathfrak{R}\right)$. Hence by Proposition 2 with the fact that the Proposition in question is valid for noetherian entire rings, $\alpha+N_{i} \in I\left(R / N_{i}\right)$, and $\alpha+\mathfrak{R} \in Q(R / \mathfrak{N})$ is contained in $I(R / \mathfrak{R})$. By Proposition 4 the injection is surjective, and $\alpha \in I R+Q \Re=I R$.

Let $R$ be a noetherian ring, let $\alpha \in R$ be regular, and let $\mathfrak{P}$ be an isolated prime ideal of $(\alpha)=R \alpha$. Let $N$ be a minimal prime ideal contained in $\mathfrak{P}$. Then $\mathfrak{F} / N$ is an isolated prime ideal of $(\alpha+N)$, and $\mathfrak{B} / N$ is of height one in $R / N$ ([4] vol. I, Th. 29, p. 238). The valua- 
tions of $R$ centered at $\mathfrak{P}$ consist of a finite number of prime divisors of $R$. A prime ideal $\mathfrak{P}$ of $R$ is of height one if for each zero prime ideal $N \subset \mathfrak{P}, \mathfrak{P} / N$ is of height one in $R / N$.

PROPOSITION 6 (THE PRINCIPAL IDEAL THEOREM). Let $\alpha$ be a regular element of a noetherian ring $R$. The isolated prime ideals of $(\alpha)$ are of height one. Conversely, if $\mathfrak{B}$ is a prime ideal of height one of $R$, there is a regular element $\alpha$ of $R$ such that $\mathfrak{P}$ is an isolated prime ideal of $(\alpha)$.

A divisorial prime ideal of $R$ is the center of some prime divisor of $R$. If $\mathfrak{A}$ is an ideal of $R$ and if $\mathfrak{P}$ is a divisorial prime ideal which is also an associated prime ideal of $\mathfrak{A}$, call $\mathfrak{P}$ a divisorial prime ideal of $\mathfrak{A}$. A prime ideal $\mathfrak{B}$ of $R$ is normal if $R_{\mathfrak{P}}$ is normal. (A ring $R$ is normal if $R=I R$.)

Proposition 7. Let $R$ be a nonimbedded noetherian ring. If $\mathfrak{P}$ is a normal prime ideal of $R$, then $\mathfrak{P}$ contains a unique zero prime ideal of $R$.

Proof. Let $N_{1}, \cdots, N_{t}$ be the distinct zero prime ideals of $R_{\Re}$. $R_{\Re}=R_{1} \oplus \cdots \oplus R_{t}$ (Prop. 3), $R_{\Re}$ is local, and therefore $t=1$.

Note that a normal divisorial prime ideal of $R$ is of height one in $R$.

4. Factorial rings, Let $R$ be a commutative ring with identity. A regular element $\alpha$ of $R$ is irreducible if $\alpha=\beta \gamma$ implies either $\beta$ or $\gamma$ is a unit of $R$. $R$ is factorial if every regular element of $R$ is a product of irreducible elements of $R$ and if $(\alpha)$ is a prime ideal of $R$ for every irreducible element $\alpha$ of $R$.

Proposition 8. If $R$ is factorial then $R$ is normal.

The Proof is as usual.

Proposition 9. Let $R=R_{1} \oplus R_{2}, R$ is factorial if and only if $R_{1}$ and $R_{2}$ are factorial.

The Proof is an application of the definition.

Proposition 10. Let $R$ have a unique zero prime ideal $\mathfrak{R} . \quad R$ is factorial if and only if $R / \mathfrak{N}$ is factorial and $\mathfrak{N}=Q \Re$.

Proposition 11. Let $R$ be a noetherian ring. $R$ is factorial if and only if every prime ideal of height one of $R$ is principal. 
The Proof is the same as for integral domains, using the principal ideal theorem (Prop. 6).

5. Finiteness conditions on $I R$ as an $R$ module. For an overring $S$ of $R$ let $\mathbb{C}(R, S)$ be $R:{ }_{R} \bar{R}$, the conductor of $R$ in $S$, where $\bar{R}$ is the integral closure of $R$ in $S$. Let $\mathfrak{N}$ be the nil radical of $R$.

Proposition 12. Let $R$ be a finitely generated ring over a field. $I R$ is a noetherian $R$ module if and only if $\mathfrak{R}=Q \mathfrak{Q}$.

Proof. $(\Rightarrow)$ : Let $d \in \mathbb{E}(R, I R)$ be regular, let $\alpha \in \mathfrak{N}$, and let $\beta \in R$ be regular. $\alpha / \beta=d(\alpha / d \beta) \in R$. $\quad(\Longleftrightarrow)$ : Let $N_{1}, \cdots, N_{t}$ be the minimal prime ideals of $R . I\left(R / N_{i}\right)$ is a noetherian $R$, or $R / N_{i}$, module ([4] vol. I., Th. 9, p. 267). $I(R / \mathfrak{R}) \simeq \bigoplus_{1=1}^{t} I(R / \mathfrak{R}) / I\left(N_{i} / \mathfrak{R}\right)$ (Prop. 3), $I(R / \mathfrak{R}) /$ $I\left(N_{i} / \mathfrak{R}\right) \simeq I\left(R / N_{i}\right)$ (Prop. 2), and $I(R / \mathfrak{R})$ is a noetherian $R$ module. $i: I R / I \mathfrak{R} \rightarrow I(R / \mathfrak{R})$ is injective, so $I R$ is a noetherian $R$ module.

Proposition 13. Let $R$ be a noetherian ring which has a unique zero prime ideal. If $I R$ is a noetherian $R$ module then $R$ is either reduced or artinian.

Proof. First assume $R=I R$. Suppose $n \in R$ is nilpotent and nonzero. Let $\alpha \in R$ be regular. For some integer $m$ and some $\beta \in R, \beta\left(n / \alpha^{m}\right)=n / \alpha^{m+1}$. Thus $n=\alpha \beta n, 1-\alpha \beta$ is nilpotent, $\alpha$ is a unit of $R$, and $R$ is artinian. For the general case, $I R$ is either reduced or artinian. If $I R$ is reduced, so is $R$. If $I R$ is artinian then $I R=Q R$, by hypothesis $\mathfrak{S}(R, I R)$ contains a regular element, and thus $R=I R$ is artinian.

Proposition 14. Let $R$ be a finitely generated ring over a field, and let $R$ have a unique zero prime ideal. $I R$ is a noetherian $R$ module if and only if $R$ is either reduced or artinian.

ExAmple. $\quad R=k[x, y]_{(x, y)} /\left(x\left(x^{2}-y^{3}\right), y\left(x^{2}-y^{3}\right)\right)$ is imbedded at $(x, y)$, is normal, and is neither reduced nor artinian. $R$ is factorial, but $R / \Re=k[x, y] /\left(x^{2}-y^{3}\right)$ is not factorial.

6. Imbedded prime ideals of proper principal ideals, Let $R$ be a noetherian ring. A proper principal ideal is a principal ideal of $R$ generated by a regular nonunit of $R$. An imbedded prime ideal of an ideal $\mathfrak{A}$ is an associated prime ideal of $\mathfrak{A}$ which is not isolated.

THEOREM 1. Let $\mathscr{D}$ be the set of all prime ideals of $R$ which occur as an embedded prime ideal of a proper principal ideal. Let 
$\alpha$ be a regular nonunit of $R$. The following are equivalent

(a) $\mathfrak{P}$ is an imbedded prime ideal of $(\alpha)$.

(b) $\mathfrak{P} \in \mathscr{D}$ and $\alpha \in \mathfrak{P}$.

Proof. See [4] vol. II, Appendix 6 for the definitions of grade and prime sequence. Suppose that $\mathfrak{P} \in \mathscr{D}$ and $\alpha \in \mathfrak{P}$. There is a regular nonunit $\beta$ of $R$ such that $\mathfrak{P}$ is an imbedded prime ideal of $(\beta)$. Hence grade $\left(R_{\Re}\right)=1$. If $\mathfrak{F}$ were not an imbedded prime ideal of $(\alpha), R_{\Re} / R_{\Re} \cdot \alpha$ would contain a regular nonunit, and grade $\left(R_{\Re}\right)$ would be greater than one.

7. Complete intersections in a ring. Let $R$ be a commutative ring with identity. A regular element $f$ of $R$ is a complete intersection in $R$ if and only if $I R \cdot f \cap R=R f$. Equivalently if $g \in R$ and $g / f \in I R$ then $g / f \in R$. For a noetherian ring $R$, let $\mathscr{A} \mathfrak{A}$ denote the set of associated prime ideals of an ideal $\mathfrak{U}$ excluding the prime ideal $R$ (so that $\mathscr{A} R=\varnothing$ ).

THEOREM 2. Let $R$ be a nonimbedded ring such that $I R$ is a noetherian $R$ module. (Then $R$ is noetherian. Let $\mathscr{C}=\bigcap_{d} \mathscr{A}(d)$ where the intersection is over all regular elements $d$ of $\mathbb{C}(R, I R)$.

(a) $\mathscr{C}=\mathscr{D} \cup \mathscr{A} \mathbb{C}(R, I R)$. Thus $\mathscr{D}$ is a finite set. (The complement of $\mathscr{D}$ in $\mathscr{C}$ consists of the prime ideals in $\mathscr{C}$ of height one, and $\mathscr{C}$ contains the nonnormal divisorial prime ideals of $R$.)

(b) Let $f$ be a regular element of $R$. The following are equivalent.

1. $f$ is a complete intersection in $R$.

2. $f$ is contained in no element of $\mathscr{C}$.

3. The divisorial prime ideals of $(f)$ are normal, thus isolated, and $(f)$ is unmixed.

The Proof follows. Let $\sqrt{ }$ denote $\mathfrak{\subseteq}(R, I R) . \quad I R_{\Re}=R_{\Re} \cdot I R([1])$.

Lemma 1. Let $d \in \mathbb{C}$ be regular, and let $f \in R$ be regular. If $f$ is contained in no element of $\mathscr{A}(d), f$ is a complete intersection in $R$.

Proof. Let $g \in R$ with $g / f \in I R$. Then $d(g / f)=c \in R$, so $c \in(d):(f)$ $=(d)$, and $g / f=c / d \in R$.

Lemma 2. If $f$, is a complete intersection in $R$, then $f$ is contained in no element of $\mathscr{A}$ (5).

Proof. Let $c \in \mathfrak{E}:(f)$. Then $c f(I K) \subset R$, so $c \in \mathfrak{E}$, and $\mathfrak{b}:(f)=\mathfrak{F}$. 
Lemma 3. If $d \in \mathfrak{C}$ is regular, then $\mathscr{A} \mathfrak{C} \subset \mathscr{A}(d)$.

Proof. Let $\mathfrak{B}$ be a prime ideal of $R, \mathfrak{P} \notin \mathscr{A}(d)$. If $\mathfrak{P}$ is a zero prime ideal, then $\mathfrak{P} \notin \mathscr{A} \mathbb{C}$. Let $f \in R_{\Re} \mathfrak{P}$ be regular with $R_{\Re} d: R_{\Re} f=$

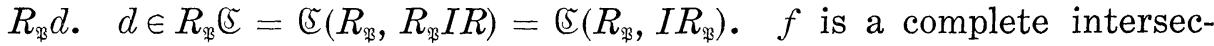

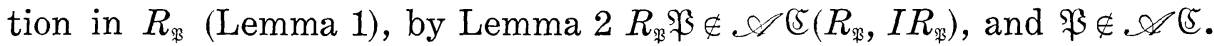

Lemмa 4. Let $f$ be a complete intersection in $R$, and let $g$ be a regular element of $R$ with $f$ contained in no divisorial prime ideal of $(g)$. Then $f$ is contained in no element of $\mathscr{A}(g)$.

Proof. Let $c \in(g):(f)$. For some $h \in R, c f=g h$, and $v(f) \leqq v(h)$ for every prime divisor $v$ of $R$. Then $h / f \in I R$ (Prop. 5), $h / f \in R$, and $c \in(g)$.

Lemma 5. If $\mathfrak{P}$ is a nondivisorial associated prime ideal of a principal ideal generated by a regular element, then $\mathfrak{P} \in \mathscr{C}$.

Proof. Let $\mathfrak{P}$ be a prime ideal containing a regular element with $\mathfrak{P} \notin \mathscr{C}$, and let $g$ be a regular element of $R$. By Lemma 1 there is a complete intersection $f$ in $R_{\mathfrak{s}} \mathfrak{P}$ contained in no divisorial prime ideal of $R_{\Re} g$. Apply Lemma 4 .

Lemma 6. Let $\mathfrak{S}$ be a divisorial prime ideal of $R$. If $\mathfrak{P}$ contains a complete intersection of $R$, then $\mathfrak{P}$ contains a unique zero prime ideal (and thus $\mathfrak{P}$ is of height one in $R$ ).

Proof. Let $f \in \mathfrak{P}$ be a complete intersection in $R$, let $N$ be a minimal prime ideal of $R$ contained in $\mathfrak{P}$ with $\mathfrak{P} / N$ of height one in $R / N$, and let $v_{1}, \cdots, v_{s}$ be the distinct prime divisors of $R$ centered at $\mathfrak{P}$ with $v_{i}(N)=\{\infty\}$. If there is a zero prime ideal distinct from $N$ contained in $\mathfrak{B}$, it follows that there is a minimal prime ideal distinct from $N$ contained in $\mathfrak{P}$. Let $N_{1}=N, N_{2}, \cdots, N_{t}$ be the distict minimal prime ideals of $R$ which are contained in $\mathfrak{P}$. Let $g \in$ $N_{2} \cap \cdots \cap N_{t}$ and $g \notin N_{1}$. Let $m_{i}=v_{i}(g)>0$ and let $n_{i}=v_{i}(f)$ for $i=1, \cdots, s$. By raising $g$ to a power $m_{i} \geqq n_{i}$ for $i=1, \cdots, s$ can be assumed. Assume $m_{1} / n_{1} \geqq m_{i} / n_{i}$ for $i=2, \cdots, s$, and let $m=m_{1}$, and $n=n_{1}$. A factor may be introduced into $g$ without destroying the above so that for all prime divisors $w$ of $R$ with $N_{w} \cap R \neq$ $N_{1}, \cdots, N_{t}, w(g) \geqq m w(f)$. Now for all prime divisors $w$ of $R, w\left(f^{m}\right) \leqq$ $w\left(g^{n}\right)$, so $g^{n} / f^{m} \in I R$ (Prop. 5), and $g^{n} / f^{m} \in R$. $v_{1}\left(g^{n} / f^{m}\right)=0$. However, if $w$ is a prime divisor of $R$ with $N_{w} \cap R=N_{2}$, then $w\left(g^{n} / f^{m}\right)=\infty$, so $g^{n} / f^{m} \in N_{2} \subset \mathfrak{P}$ which is a contradiction. 
Lemma 7. Let $d \in \mathfrak{S}$ be regular. If a divisorial prime ideal $\mathfrak{P}$ is not an associated prime ideal of $(d)$, then $\mathfrak{P}$ is normal.

Proof. $d \in \mathbb{E}\left(R_{\Re}, I R_{\Re}\right)$, so in $R_{\Re}$ there is a complete intersection $f \in R_{\mathfrak{B}} \mathfrak{P}$. $\mathfrak{P}$ is of height one $R$ (Lemma 6), and $\mathfrak{S} \not \subset \mathfrak{P}$.

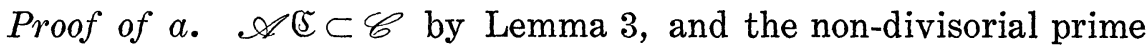
ideals in $\mathscr{D}$ are in $\mathscr{C}$ by Lemma 5 . A divisorial prime ideal in $\mathscr{D}$ is nonnormal by Prop. 7 , and by Lemma 7 it is in $\mathscr{C}$. Thus $\mathscr{D} \cup$ $\mathscr{A} \subset \mathscr{C} \subset \mathscr{C} \cup \mathscr{D} \cup \supset \mathscr{C}$ for an isolated prime ideal of a principal ideal is of height one, and the height one prime ideals in $\mathscr{C}$ are in $\mathscr{A C}$. The last assertion is Lemma 7.

Proof of b. $(1 \Rightarrow 2)$ : By Lemmas 6 and 2 there is a regular element $d$ of $\sqrt{5}$ which is contained in no divisorial associated prime ideal of $(f)$. Apply Lemma 4. $(2 \Rightarrow 3)$ : If $\mathfrak{P}$ is a divisorial associated prime ideal of $(f) \mathfrak{B}$ is normal (Lemma 7). $(f)$ is unmixed (Lemma 5). $(3 \Rightarrow 1):(f): \mathbb{}=(f)$. If $g \in R$ and $g / f \in I R$, then $g \mathbb{} \subset(f)$, and $g / f \in R$.

Proposition 15. Let $R$ be a commutative nonnull ring with identity, and let $f$ and $g$ be complete intersections in $R$. Then $f g$ is a complete intersection in $R$, and if $f / g \in R$ it is a complete intersection in $R$. Let $f \in R$ be such that $f^{n}$ is a complete intersection in $R$ for some integer $n \geqq 1$. Then $f$ is a complete intersection in $R$.

Proof. Let $h \in R$. If $h / g f \in I R$ then $h / g \in I R, h / g \in R$, and $h / g f \in R$. If $g / f \in R$ and $h f / g \in I R$, then $h f / g \in R$. If $h / f \in I R$, then $h f^{n-1} / f^{n} \in I R$ and $h / f \in R$.

Let $R$ be a nonimbedded noetherian ring, and let $D$ be the divisor group of $R$, the free abelian group generated by the prime divisors of $R(\S 3)$. If $f$ is a regular element of $Q R$, let $(f)=\sum_{v} v(f) \cdot v$ where the sum is over all prime divisors $v$ of $R$. Let $P=\{(f) \mid f \in Q R, f$ regular\}, the subgroup of $D$ of principal divisors, and let $I$ be the subgroup of $P$ generated by $\{(f) \mid f$ is a complete intersection in $R\}$. $I=P$ if and only if $R=I R$, and thus $I=D$ if and only if $R$ is factorial (Prop. 11). $P / I$ is torsion free (Prop. 15).

Let $\mathfrak{A} \in D$ be a complete intersection, that is, let $\mathfrak{A} \geqq 0$ and $\mathfrak{U} \in I$. Then (Prop. 15) there is a complete intersection $f$ in $R$ with $\mathfrak{U}=(f)$. If $h \in R,(h) \in I$ if and only if $h$ is a complete intersection in $R$.

8. Examples. 1. Let $R$ be a reduced finitely generated ring over a field, of height one. The complete intersections in $R$ are those regular elements of $R$ which do not vanish at the nonnormal (singular) 
primes of $R$. E.g., for the plane curve $y^{2}-x^{3}, P / I=Z$, the integers, and for $y^{2}-x^{3}-x^{2}, P / I=Z \oplus Z$.

2. Let $R$ be the coordinate ring of a reduced complete intersection in affine space. Then $\mathscr{C}$ contains only prime ideals of height one of $R$. For $R$ is Cohen-Macaulay, and in particular every proper principal ideal is unmixed. E.g., the surfaces $y^{2}-x^{3}, y^{2}-x^{3}-z x^{2}$ in affine 3 -space.

3. Let $R=k\left[t^{4}, t^{3} u, t u^{3}, u^{4}\right] \subset k[t, u]$ the polynomial ring. $I R=$ $k\left[t^{4}, t^{3} u, t^{2} u^{2}, t u^{3}, u^{4}\right]$. The two affine localizations $k\left[1, u, u^{3}, u^{4}\right]$ and $k\left[t^{4}, t^{3}, t, 1\right]$ of $R$ are integrally closed. Hence $\mathscr{C}=\left\{\left(t^{4}, t^{3} u, t u^{3}, u^{4}\right)\right\}$.

4. Let $\left.R=k\left[t^{6}, t^{4} u^{2}, t^{3} u^{3}, t u^{5}, u^{6}\right)\right] \subset k[t, u]$.

$$
I R=k\left[t^{6}, t^{5} u, t^{4} u^{2}, t^{3} u^{3}, t^{2} u^{4}, t u^{5}, u^{6}\right],
$$

and

$$
\mathfrak{C}=\left(t^{4} u^{2}, t^{3} u^{3}, t u^{5}, u^{6}\right)=\mathfrak{P}
$$

a prime ideal. $t^{6} \notin \mathfrak{P},\left(t^{4} u^{2}\right)^{2} / t^{6} \in I R, \notin R$, so $\left(t^{6}\right)$ is imbedded at $\mathfrak{M}=$ $\left(t^{6}, \mathfrak{P}\right)$, and $\mathscr{C}=\{\mathfrak{P}, \mathfrak{M}\}$. Thus $\mathscr{A} \mathbb{C}$ may be strictly less than $\mathscr{C}$.

5. Let $R=k[x, y, z] /(z x, z y) . \quad(x, y, z)$ is the only nonnormal prime ideal of $R$, and it is divisorial.

6. Let $R$ be a reduced, finitely generated ring over a field $k$. Then there exist $f_{1}, \cdots, f_{s} \in R$ such that $\left(f_{1}, \cdots, f_{s}\right)$ is of codimension $s$ in $R$, is unmixed and self radical. Induct on $s$. For $s=1$, choose $f_{1}$ such that the locus of $f_{1}$ is $k$-transversal to Spec $R$, and such that $f_{1}$ is contained in no element of $\mathscr{C}$. The step from $s-1$ to $s$ is the same as for $s=1$ on the ring $R /\left(f_{1}, \cdots, f_{s-1}\right)$. By affine localization, it follows that a projective reduced algebraic variety possesses a complete intersection of any codimension (which is locally a complete intersection of codimension $s$ given globally by $s$ forms contained in the homogeneous coordinate ring of the variety).

7. Let $R$ be a nonimbedded ring such that $I R$ is a noetherian $R$ module, let $\mathfrak{\Re} \in \mathscr{C}$, and suppose that the order function $w$ of $R_{\Re}$ ([2], vol. II, p. 249) is a valuation. Let $\mathfrak{m}=R_{\mathfrak{\beta}} \Re$. Let $f \in \mathfrak{m}$, with $f$ contained in no other element of $\mathscr{C}\left(R_{\Re}\right)$. Let $\mathfrak{q}$ be a primary component of $(f)$ associated to $\mathfrak{m}$. For some integer $n \geqq 0, \mathfrak{m}^{n+w(f)} \subset \mathfrak{q}$. Thus if $g \in R_{\Re}$, if $g / f \in I R_{\Re}$ and if $w(g) \geqq n+w(f)$, then $g / f \in R$. Let $f^{\prime} \in \mathfrak{m}$ be contained in no other prime in $\mathscr{C}\left(R_{\mathfrak{\beta}}\right)$ with $n^{\prime}$ such that $w(g) \geqq n^{\prime}+w\left(f^{\prime}\right)$ and $g / f^{\prime} \in I R_{\Re}$ imply $g / f^{\prime} \in R_{\Re}$. Let $g \in R_{\Re}$ with 
$w(g) \geqq n+w\left(f^{\prime}\right)$ and $g / f^{\prime} \in I R_{\Re}$. There is an integer $\ell \geqq 0$ such that $w\left(g f^{\prime}\right) \geqq n^{\prime}+w\left(f^{\prime}\right)$. Then $g f^{\ell} / f^{\prime} \in R_{\mathfrak{r}}$, and $g / f^{\prime} \in R_{\Re}$. Let $a(\mathfrak{F})$ be the subset of nonnegative integers $n$ such that for any $f \in \mathfrak{m}$ con-

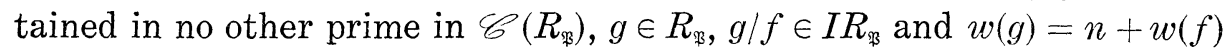
imply $g / f \in R_{\Re}$. Thus $a(\mathfrak{F})$ contains all but finitely many of the nonnegative integers. Let $f$ be an element of $m$ contained in no other prime in $\mathscr{C}\left(R_{\mathfrak{F}}\right)$. If $n \geqq 0$ is such that $g \in R_{\mathfrak{p}}, g / f \in I R_{\mathfrak{r}}$ and $w(g) \geqq$ $n+w(f)$ imply $g / f \in R_{\Re}$, and if $n+w(f) \in a(\mathfrak{P})$, then $n \in a(\mathfrak{P})$.

8. Let $R$ be noetherian and have a unique nonzero zero prime ideal. Then the complete intersections in $R$ are the units of $R$. Let $n \neq 0$ be nilpotent, and let $f$ be a complete intersection in $R$. Then $n=c f n$ for some $c \in R,(1-c f) n=0$, so $1-c f$ is nilpotent and $f$ is unit.

9. Projective algebraic varieties and o-equivalence. Let $V$ be a projective algebraic variety defined over a field $k$, such that $I k[V]$ is a noetherian $k[V]$ module where $k[V]$ is the homogeneous coordinate ring of $V$ relative to a fixed projective embedding. $C=\mathbb{C}(k[V], I k[V])$ is a homogeneous ideal of $k[V]$, and an affine localization of $[s$ is the conductor of the affine localization of $k[V]$ in its integral closure. Affine localization also preserves primary decomposition. Thus the prime ideals in $\mathscr{C}=\mathscr{C}(k[V])$ are homogeneous, $\mathscr{C}$ can be defined locally, and $\mathscr{C}$ is a biregular invariant over $k$ if the irrelevant ideal $\mathfrak{\Im}$ of $k[V]$ is ignored. Let $D$ be the group of divisors of $\boldsymbol{V}$ over $k$. $\mathfrak{X} \in D$ is a complete intersection on $V$ if and only if locally $\mathfrak{A}$ is a complete intersection and $\mathfrak{A}=(F)$ for some homogeneous element $F$ of $k[V]$. Call $F \in k[V]$ a complete intersection on $V$ if $F$ is homogeneous and if $(F)$ is a complete intersection on $V$, which is true if and only if $F$ is contained in no prime in $\mathscr{C}$ other than $\mathfrak{\Im}$ if $\mathfrak{\Im} \in \mathscr{C}$. The discussion of Example $7, \S 8$ holds for $\mathfrak{\Im}$ where degree is the order function $w$. Define $a(\boldsymbol{V})$ similarly to $a(\widetilde{\Im})$. By a Segré transformation a projective variety $V^{\prime}$ can be found which is biregularly equivalent to $V$ and for which $a\left(V^{\prime}\right)$ contains the positive integers.

Let $F \in k[V]$ be regular, homogeneous, non-constant and contained in no prime of $\mathscr{C}$ other than possibly $\mathfrak{I}$. Let $S=k[V \sim V(F)] \sim$ $\bigcup_{\mathfrak{B} \in \mathcal{P}}$ and let $\mathfrak{o}=S^{-1} k[V \sim V(F)] . \quad \mathfrak{o}$ is a biregular invariant of $V$ over $k$. Let $\mathfrak{X}, \mathfrak{B} \in D$. Define $\mathfrak{U} \rightarrow \mathfrak{B}$ if and only if $\mathfrak{B}=\mathfrak{U}+(f)$ for some $f \in \mathfrak{D}$. Define $\mathfrak{A} \sim \mathfrak{B}, \mathfrak{A}$ is $\mathfrak{0}$-equivalent to $\mathfrak{B}$, if and only if $\mathfrak{A} \rightarrow \mathfrak{B}$ and $\mathfrak{B} \rightarrow \mathfrak{U}$ which is equivalent to $\mathfrak{B}=\mathfrak{A}+(f)$ for some unit $f$ of $\mathfrak{o}$. (A unit of $\mathrm{D}$ is a quotient of two complete intersections on $V$ in $k[V]$ of equal degree.) The complete system of $\mathfrak{U} \in D$ is $|\mathfrak{U}|=\{\mathfrak{B} \in D \mid \mathfrak{B} \geqq 0$, $\mathfrak{U} \rightarrow \mathfrak{B}\} . \quad\{f \in \mathfrak{D} \mid \mathfrak{X}+(f) \geqq 0\}$ is a function module. $\mathscr{L}_{m}=\{(F) \mid F \in k[V]$ homogeneous of degree $m\}$. 
Proposition 16. For all $m$ sufficiently large there is a complete intersection on $V$ in $k[V]$ of degree $m$ and $\mathscr{L}_{m}$ is a complete system. If $k$ is infinite and algebraically closed in $k(V)$, then $\mathscr{L}_{m}$ is a complete system if and only if $m \in a(\boldsymbol{V})$.

Proof. If $k$ is infinite then there is a linear form $L \in k[V]$ contained in no prime in $\mathscr{C}$ other than possibly $\mathfrak{\Im}$. If $k$ is finite, let $p$ be a prime, and let $k_{i}$ for $i=1,2, \cdots$ be an extension field of $k$ such that $k_{i} \subset k_{i+1}$ and $\left[k_{i+1}: k_{i}\right]=p$. For some $k_{i}$, there is a linear form $L$ defined over $k_{i}$ contained in no prime in $\mathscr{C}$ other than $\Im$. The $k_{i} / k$ norm of $L$ is a complete intersection on $\boldsymbol{V}$ in $k[\boldsymbol{V}]$ of degree $p^{b}$ for some $\ell$. Using two distinct primes, it follows that there is a complete intersection of degree $m$ for all $m$ sufficiently large.

Let $m \in a(V)$, and let $F \in k[V]$ be a complete intersection of degree m. $\mathscr{L}_{m} \subset|(F)|$. Let $\mathfrak{A} \in|(F)|$. There are $H, G \in k[V]$ homogeneous of equal degree, $(G)$ a complete intersection on $V$ with $\mathfrak{A}=(H / G)+$ $(F)$. So $F H / G \in k[V]$, and $\mathscr{L}_{m}=|(F)|$.

Now suppose $k$ is infinite and algebraically closed in $k(\boldsymbol{V})$. Suppose $\mathscr{L}_{m}$ is a complete system, and let $(F) \in \mathscr{L}_{m}$ where $F$ is a complete intersection on $V$ in $k[V]$. Then $\mathscr{L}_{m}=|(F)|$. Let $G$ be regular homogeneous element of $k[V]$ contained in no prime of $\mathscr{C}$ other than Э. Let $H \in k[V]$ homogeneous with $(H) \geqq(G)$ and $\operatorname{deg} H=\operatorname{deg} G+m$. Then $(F) \rightarrow(H)-(G)$, there is an $E \in k[V]$ with $(E)=(H)-(G)$, $H / G E$ is algebraic over $k$, and $H / G \in k[V]$. Thus $m \in a(\boldsymbol{V})$.

\section{REFERENCES}

1. Joseph Lipman, Some properties of normalization and localization, Proc. Amer. Math. Soc., 16 (1965), 1120-1122.

2. M. Rosenlicht, Equivalence relations on algebraic curves Ann. of Math., 56 (1952), 169-191.

3. A. Seidenberg, The hyperplane sections of normal varieties, Trans. Amer. Math. Soc., 69 (1950), 357-386.

4. O. Zariski and P. Samuel, Commutative Algebra, Princeton, D. Van Nostrand, 1958 and 1960 .

Received November 11, 1971. The work on this paper was supported in part by National Science Foundation Grant GP-7461.

The University of Kansas 



\section{PACIFIC JOURNAL OF MATHEMATICS}

EDITORS

\author{
H. SAMELSON \\ Stanford University \\ Stanford, California 94305 \\ C. R. HOBBY \\ University of Washington \\ Seattle, Washington 98105
}

\author{
J. DUGUNDJI \\ Department of Mathematics \\ University of Southern California \\ Los Angeles, California 90007 \\ RICHARD ARENS \\ University of California \\ Los Angeles, California 90024
}

\section{ASSOCIATE EDITORS}

E. F. BECKENBACH

B. H. NeUManN

F. WOLF

K. YosHIDA

\section{SUPPORTING INSTITUTIONS}

UNIVERSITY OF BRITISH COLUMBIA

CALIFORNIA INSTITUTE OF TECHNOLOGY

UNIVERSITY OF CALIFORNIA

MONTANA STATE UNIVERSITY

UNIVERSITY OF NEVADA

NEW MEXICO STATE UNIVERSITY

OREGON STATE UNIVERSITY

UNIVERSITY OF OREGON

OSAKA UNIVERSITY
UNIVERSITY OF SOUTHERN CALIFORNIA

STANFORD UNIVERSITY

UNIVERSITY OF TOKYO

UNIVERSITY OF UTAH

WASHINGTON STATE UNIVERSITY UNIVERSITY OF WASHINGTON

AMERICAN MATHEMATICAL SOCIETY NAVAL WEAPONS CENTER 


\section{Pacific Journal of Mathematics}

\section{Vol. 45, No. $1 \quad$ September, 1973}

William George Bade, Complementation problems for the Baire classes .......... 1

Ian Douglas Brown, Representation of finitely generated nilpotent groups ........ 13

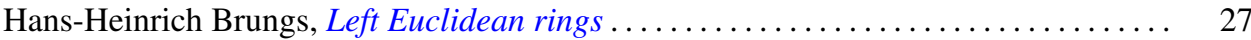

Victor P. Camillo and John Cozzens, A theorem on Noetherian hereditary rings ..... 35

James Cecil Cantrell, Codimension one embeddings of manifolds with locally flat

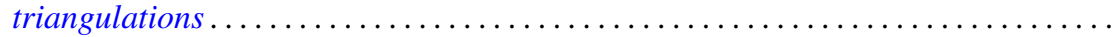

L. Carlitz, Enumeration of up-down permutations by number of rises . . . . . . . . . .

Thomas Ashland Chapman, Surgery and handle straightening in Hilbert cube

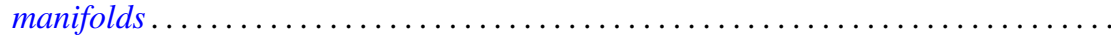

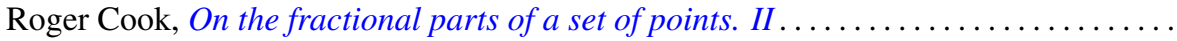

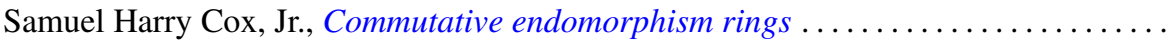

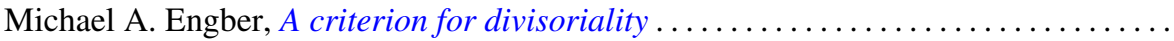

Carl Clifton Faith, When are proper cyclics injective . . . . . . . . . . . . . . 97

David Finkel, Local control and factorization of the focal subgroup . . . . . . . . . 113

Theodore William Gamelin and John Brady Garnett, Bounded approximation by

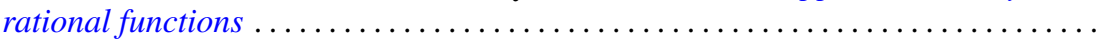

Kazimierz Goebel, On the minimal displacement of points under Lipschitzian

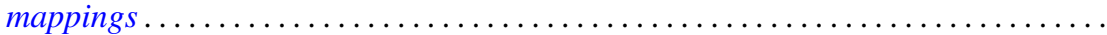

Frederick Paul Greenleaf and Martin Allen Moskowitz, Cyclic vectors for representations associated with positive definite measures: nonseparable

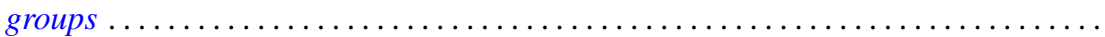

Thomas Guy Hallam and Nelson Onuchic, Asymptotic relations between perturbed linear systems of ordinary differential equations .

David Kent Harrison and Hoyt D. Warner, Infinite primes of fields and completions. .

James Michael Hornell, Divisorial complete intersections . ......

Jan W. Jaworowski, Equivariant extensions of maps ..............

John Jobe, Dendrites, dimension, and the inverse arc function .. .

Gerald William Johnson and David Lee Skoug, Feynman integrals of non-factorable

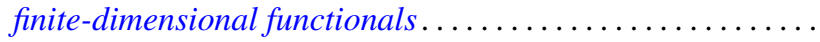

Dong S. Kim, A boundary for the algebras of bounded holomorphic functions ...... 269

Abel Klein, Renormalized products of the generalized free field and its derivatives ... 275

Joseph Michael Lambert, Simultaneous approximation and interpolation in $L_{1}$ and

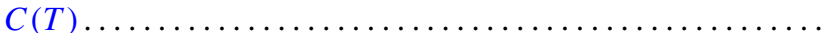

Kelly Denis McKennon, Multipliers of type $(p, p)$ and multipliers of the group

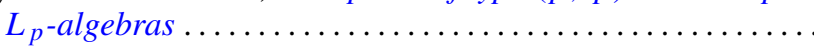

William Charles Nemitz and Thomas Paul Whaley, Varieties of implicative

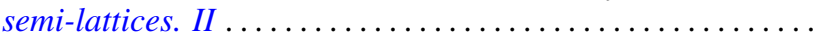

Donald Steven Passman, Some isolated subsets of infinite solvable

Norma Mary Piacun and Li Pi Su, Wallman compactifications on E-completely

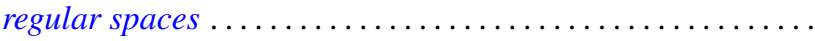

Jack Ray Porter and Charles I. Votaw, $S(\alpha)$ spaces and regular Hausdorff extensions....

Gary Sampson, Two-sided $L_{p}$ estimates of convolution transforms .

Ralph Edwin Showalter, Equations with operators forming a rig
Raymond Earl Smithson, Fixed points in partially ordered sets .

Victor Snaith and John James Ucci, Three remarks on symmetric products and

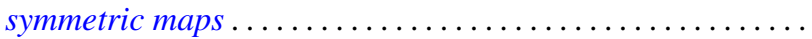

